\title{
Comparing Plastic Deformations Produced by HPT and ECAP Processes Using the Finite Element Analysis Method
}

\author{
Zeynali E. , Bisadi H. \\ Mechanical Engineering Department, Iran University of Science \& Technology, Tehran, Iran
}

\begin{abstract}
Applying Sever Plastic Deformation (SPD) to metals in order to form a nanocrystalline structure in them has been the subject of many recent researches. Investigations show that the nanocrystalline structure improves mechanical properties such as the yield strength and wear resistance. Among the SPD methods, more attention has been paid to the High Pressure Torsion (HPT) and Equal Cannel Angular Pressing (ECAP) methods. Irrespective of different work-piece geometries produced through these methods, studying the amount of plastic deformation as well as the method of applying it could be useful in comparing these processes and choosing the more effective ones. In this article, the results of finite element analysis of the HPT and ECAP processes on pure commercial aluminum are provided and the rate of success of each process in applying the SPD to the part is studied. Plastic deformation is considered as a parameter for calculating the amount of grain refinement. The two methods are compared and their advantages and disadvantages are discussed in the end.
\end{abstract}

Keywords HPT, ECAP, nanocrystal, finite element, plastic deformation

\section{Introduction}

The grain size in polycrystalline materials plays a very important role in their mechanical strength and wear resistance. In general, a fine-grained material structure has many advantages over a coarse-grained one, and besides improving the mechanical properties, it helps raise the possibility of applying superplastic deep drawing processes to that material [1-6]. Such structures in polycrystalline materials are known as Ultra Fine Grained (UFG) $[7,8]$. UFG materials are divided into microcrystal and nanocrystal groups, and in the latter group, the size of the grains is reduced to $200 \mathrm{~nm}$. The two main methods for making UFGs are the bottom-up and the top-down methods[9]. In the bottom-up method, the bulk is made through the merging of atoms or solid nanometric particles. Like the inert gas condensation[10] and electro deposition[11] methods. These methods too, have the ability to produce materials with desired grain refinements; however, they have their own disadvantages and flaws as well. One flaw is the high risk of pollution infiltration, while the other is the inevitability of residual porosity. But in the top-down methods, there are no problems of pollution and porosity. In these methods, the initially coarse-grained work-pieces go through the relevant process and the desired

* Corresponding author:

Ebrahim_Zeynali@mecheng.iust.ac.ir (Zeynali E.)

Published online at http://journal.sapub.org/mechanics

Copyright (C) 2012 Scientific \& Academic Publishing. All Rights Reserved grain refinement is achieved. There are several methods in this category, and all of them are based on imposing high deformation upon the material. The top-down methods are generally known as severe plastic deformation (SPD) processes[12].

\section{The SPD Processes}

The SPD is a process in which the formation of the desired metal is achieved through applying high hydrostatic pressure. The initial metal part is subjected to high deformation, and the desired grain refinement is achieved, without changing the overall dimensions of the initial workpiece. Among the SPD methods, more attention has been paid to the HPT and ECAP processes[13-15].

\subsection{The ECAP Process}

So far, many useful investigations have been conducted by researchers on the ECAP process. In Fig. 1, the principles of the ECAP process are shown[13].

In this figure, the conduit angle $\Phi$ and the bend angle $\psi$ are shown. Both of these angles play an important role in the process. The workpiece in this case is a rod or a wire which is placed in the conduit and then is forced into the die with the help of a punch press. The workpiece deforms by means of simple shear strain that accumulates and builds up when the workpiece passes the edges of the die. In practice, for simplifying the process, the angle of conduit is set at 90 degrees. 
The bend angle has a lesser effect on the amount of applied deformation, and in detachable dies it could be disregarded; however, in fixed and non-detachable dies, some bend angle is inevitable. Although, severe plastic deformation is applied while the workpiece passes through the shear plane, the sample leaves the die without any changes on its cross sectional area. Therefore, it could be placed within the die again and go through another pass of the process. In practice, repetition of several passes is common in order to increase the applied plastic deformation. Therefore, in each pass, relative to the previous one, the samples are rotated 90 to 180 degrees around the axis normal to the cross sectioned area [13].

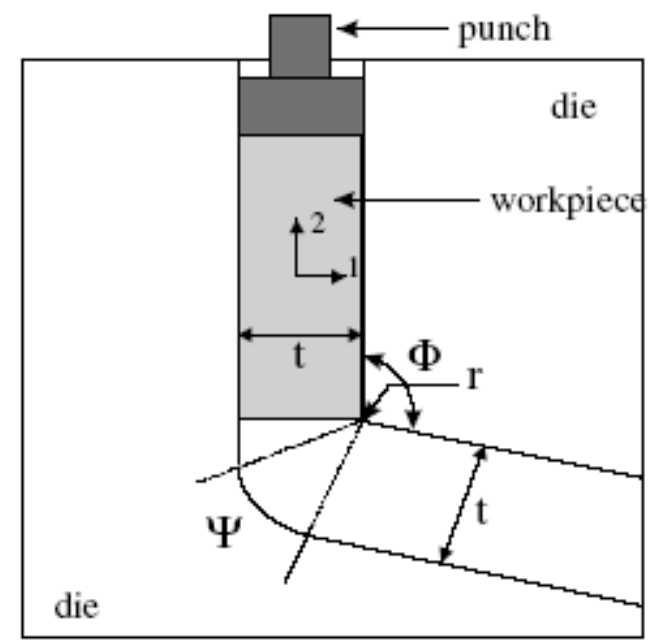

Figure 1. The schematic principles of the ECAP process[13]

\subsection{The HPT Process}

In this method, while the workpiece is under a high axial pressure, it is subjected to a moment as well; so, a severe shear strain is applied to the material. This deformation would finally lead to a grain refinement of nanometric scale. Fig. 2 shows a schematic view of this process[16].
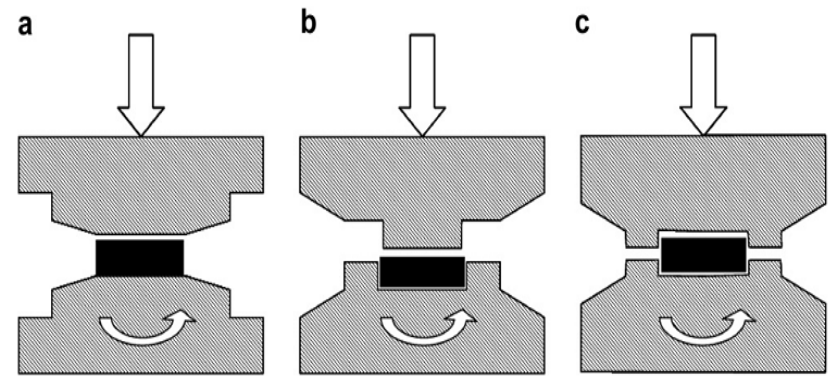

Figure 2. schematic principle of the HPT process [16]

In Fig. 2(a), the workpiece does not adhere to any side surface and can flow freely. This method can be used in some cases, where relatively lower pressure and rotation are needed. Hydrostatic pressure is very low in these dies and grain refinement is achieved as a result of friction between the surfaces of the workpiece and the die. When higher grain refinement is required and the workpiece is under high pressure and requires numerous rotations, the following two methods are more effective. In Fig. 2(b), the workpiece is placed within the cavity of the bottom anvil and is fully constrained. In Fig. 2(c), the semi-constrained workpiece is placed within the die, and during the process, parts of the workpiece metal is squeezed out of the die through its parting line as flash. The friction between the flash and the surface of the die creates the required hydrostatic pressure[16]. Nanometric grain refinement in metals improves their mechanical properties. The Hall-Petch relation verifies this fact by relating the shear strength of metals to the mean size of grains $[17,18]$.

$$
\sigma_{y_{s}}=\sigma_{o}+k_{y} d^{-\frac{1}{2}}
$$

In this relation, $\sigma_{o}$ is the friction stress, $k_{v}$ is the constant of yielding, and $d$ is the grain size.

According to this relation, it becomes clear that the yield stresses of the materials resulting from the HPT are high, because the grains are refined at nanometric levels as a result of this process. In the HPT process, hydrostatic pressure is increased up to $5 \mathrm{GPa}$ and even to $7 \mathrm{GPa}$ in some reports. Due to such high pressures, the workpieces producible in the HPT method are relatively small, and also owing to the nature of the process, they have an axisymmetrical geometry. The HPT process is used more for producing discs with a diameter of about $10 \mathrm{~mm}$ and a thickness of $1 \mathrm{~mm}$, and rings with an equal surface area. In Fig. 3, the geometry of the workpiece is shown, depicting the deformation applying angle and the parameters that are used for calculating the deformation[19].

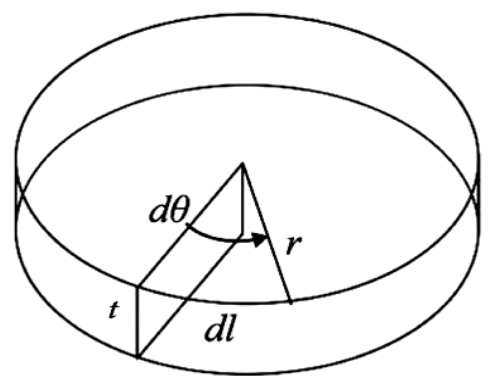

Figure 3. Deformation applying angle and the parameters for calculating the deformation[19]

Due to the axisymmetrical nature of the HPT process, the applied deformation is not homogeneous all across the radial direction, but varies according to the following relation[20, 21]:

$$
\gamma(r)=\frac{2 \pi n r}{t}
$$

In this relation, $t$ is the thickness of the workpiece, $r$ is the radial position and $n$ is the number of fully completed rotations applied to the workpiece.

As a result of this nonhomogeneous deformation, the nanostructure and the final hardness of the workpiece, vary according to the radial position at different points. The relative nonhomogeneity in the structure of the HPT disc has been verified by consulting the results of scientific tests in various reports. Fig. 4 illustrates the results of hardness measurements for a disc along the radial positions[22]. 


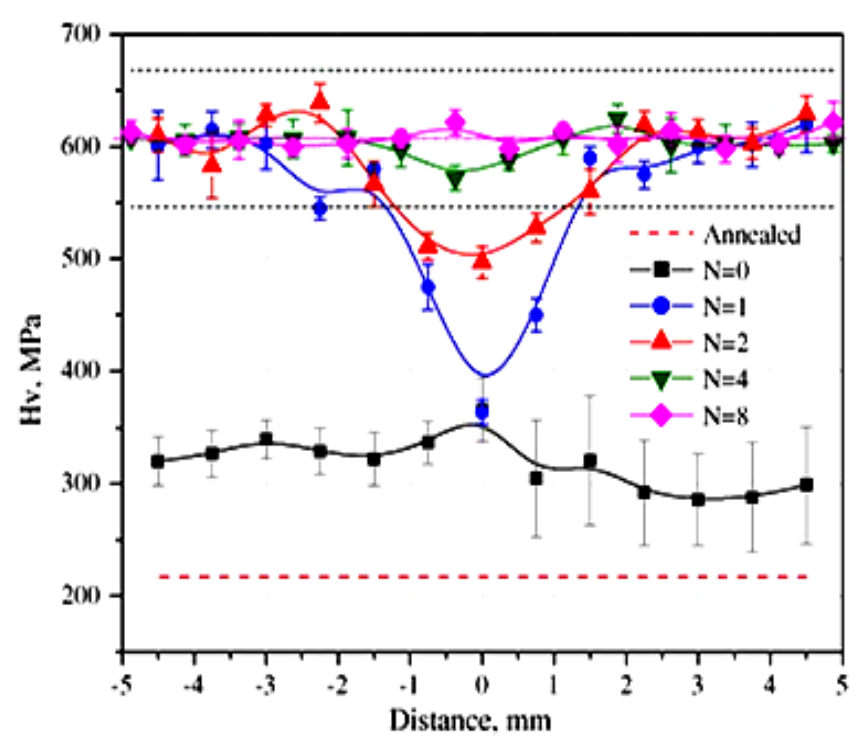

Figure 4. the results of hardness measurement for a disc along the radial positions[22]

In the HPT process, one of the main challenges for researchers is to overcome the nonhomogeneous structure of the material. Increasing the applied pressure and the number of completed rotations may enhance the homogeneity of these workpieces[22].

\section{Finite Element Simulation}

When it comes to the geometry and dimensions of workpieces, the ECAP process is more versatile and accommodating than the HPT process. In this article, a cylinder with a diameter of $20 \mathrm{~mm}$ and a length of $10 \mathrm{~cm}$ was used as an ECAP workpiece, and a disc with a diameter of $20 \mathrm{~mm}$ and a thickness of $5 \mathrm{~mm}$ was used to simulate the HPT process. The finite element analysis was performed using the commercially available software "DEFORM 3D" and triangular elements were used to simulate both processes. Both workpieces were made of pure commercial aluminum. The meshed workpieces are shown in Fig. 5.
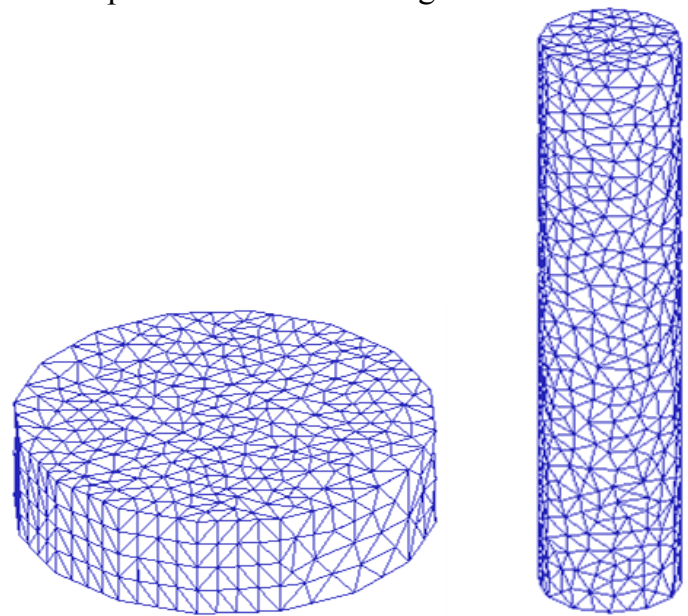

Figure 5. Meshed workpieces for the simulation of ECAP and HPT processes

\subsection{The ECAP Process Simulation}

In order to simulate the ECAP process, a die with a conduit angle of 90 degrees and a bend angle of 0 degree was used. The punch's movement speed within the conduit was $1 \mathrm{~mm} / \mathrm{sec}$. The workpiece went through one ECAP pass and the simulation process was stopped once the entire workpiece had passed by the conduit angle. The simulation results of the ECAP process are shown in Fig. 6. Changes in the amount of effective strain in the workpiece, throughout the ECAP process, are shown in Figs. 6(a) to 6(d). In Fig. 6(a) only a small portion of the workpiece has passed the conduit angle, and in Fig. 6(d), the entire workpiece has experienced one ECAP pass. As shown in this figure, plastic deformation is applied as the workpiece passes by the conduit angle. After passing through this area, deformation remains almost contant at every point of the workpiece. Although, as a result of friction with the conduit wall, a small amount of strain may be produced on the workpiece surface as it moves through the conduit, but this strain could be disregarded. According to the simulation results, the amount of strain produced from one ECAP pass is about 1.5, and in order to increase the effective strain, the workpiece must be placed within the conduit again and the process must be repeated.

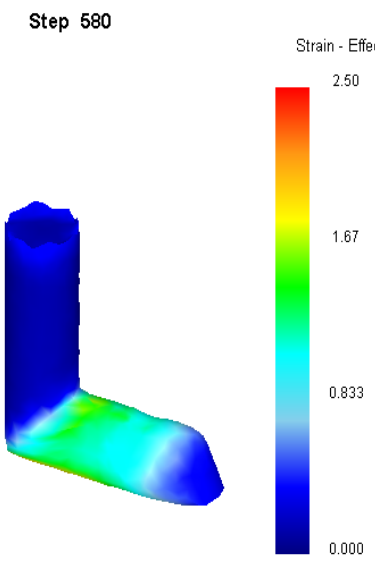

(a)

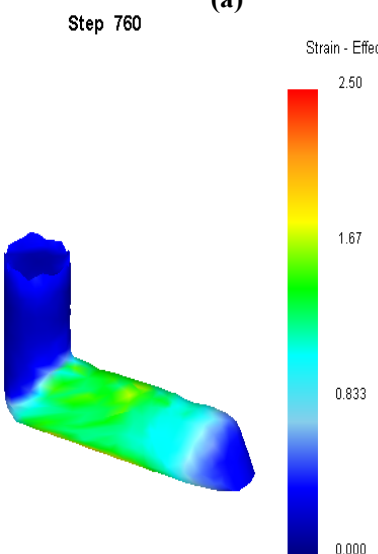

(c)
Step 420

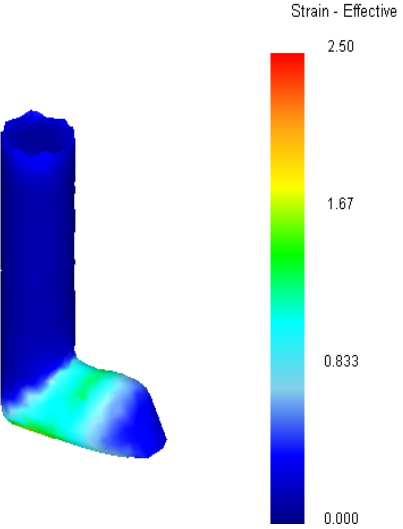

(b)

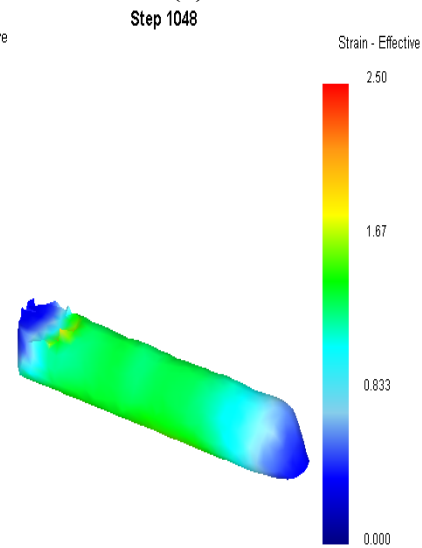

(d)
Figure 6. Simulation results of the ECAP process

\subsection{The HPT Process Simulation}

The HPT process simulation is carried out on a semi- 
constrained workpiece. Fig.7 shows the deformation of the workpiece during the process as well as the amount of effective strain at every point of the workpiece.

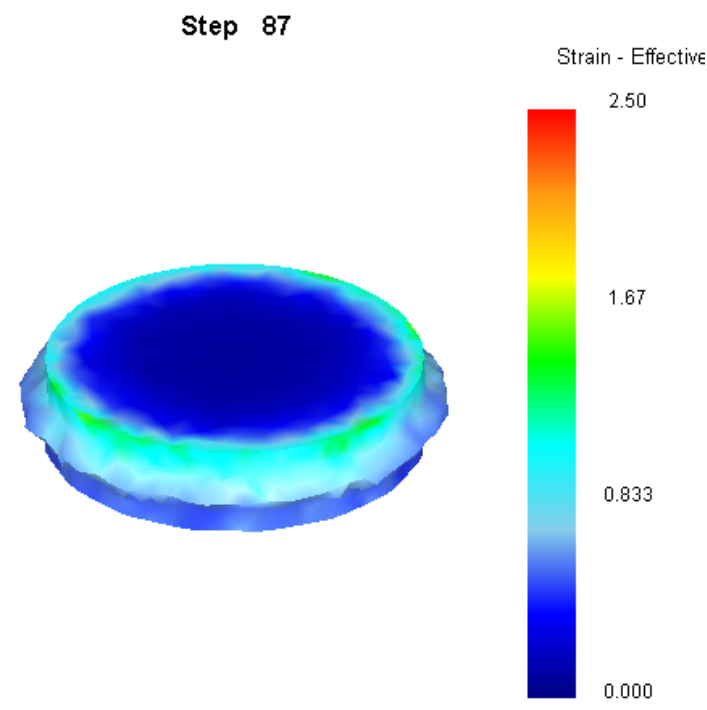

(a)

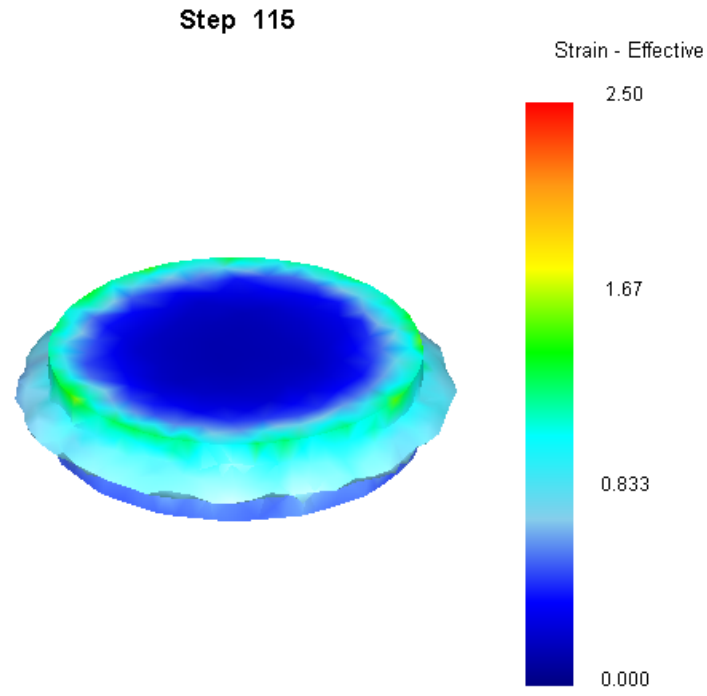

(b)

Step 228

Strain - Effective

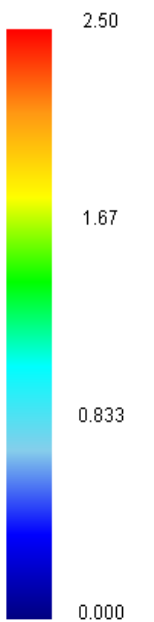

(c)
Step 278

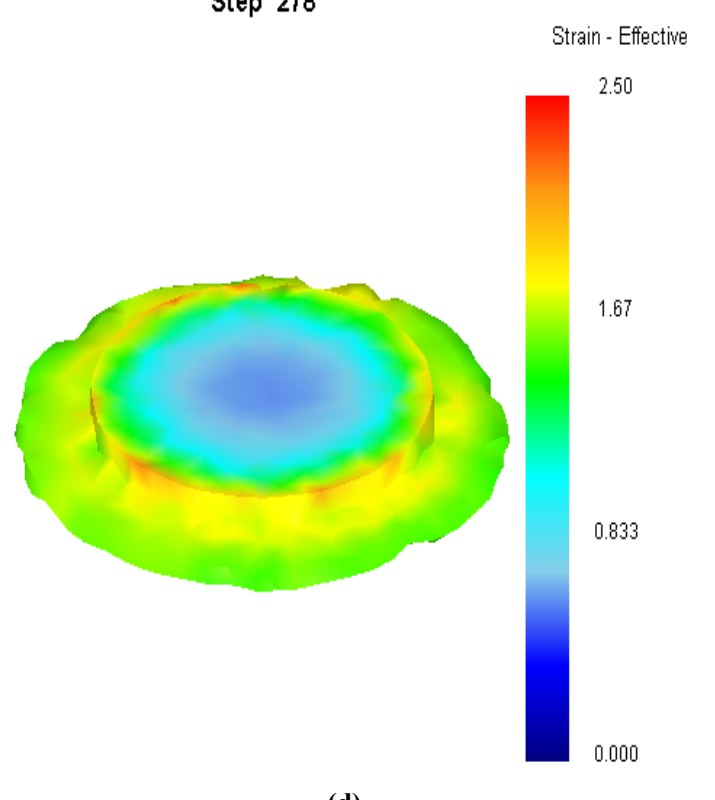

(d)

Figure 7. Deformation of the workpiece during the HPT process

As shown in Fig. 7(a) to 7(d), some flash is formed in the die parting line, which increases as the process continues. According to the simulation results, and consistent with the existing experimental reports[3-7], as the process continues and the number of rotations increases, the plastic deformation at every point of the disc continuously increases. In Fig. 7, there is an obvious deformation gradient on the HPT workpiece, and it could be seen that the points closest to the outer edge of the workpiece always go through more plastic deformation. The other important conclusion that could be made from the results of this simulation is that the amount of effective strain obtained after 4 rotations in the HPT process, 2.5 on the outer edge of the workpiece, is higher than the effective strain resulting from the ECAP process after one pass. In this HPT simulation, a vertical pressure of $2 \mathrm{GPa}$ is applied to the workpiece, and the rotation is applied via the lower holder, with a speed of $1 \mathrm{rpm}$. Since increasing the number of rotations in the HPT process is much easier than repeating the passes in the ECAP process, it could be said that applying plastic deformation in the HPT process is much easier and faster than in the ECAP process. On the other hand, if flash production could be curtailed so that anvils don't rub against each other as a result of high pressure, obtaining more deformations would be easier. In order to avoid the rubbing of holders, the pressure in this research did not exceed $2 \mathrm{GPa}$. The other point in the HPT process that must be considered is the significant difference between the amount of deformation resulting from relation (2) and the amount of actual deformation at the end of the process; as the amount of actual deformation is much less than what is predicted by the theory. The reason for this difference should be sought in the phenomena of relative slipping of the workpiece and the pressure surface of the die. Indeed, what happens in practice is a sliding friction, which results in the transfer of only a part of the total rotation of the die to the workpiece. 


\section{Discussion and Conclusions}

According to the results of the finite element simulations, the HPT and ECAP processes could be compared to one another from three aspects:

\section{1) The amount of plastic deformation}

Because of the high pressure applied in the HPT process, and the continuous circular deformation, the amount of plastic deformation caused by the HPT process is more than the ECAP process. Therefore, it could be concluded that more grain refinement is obtained in metals through the HPT process.

\section{2) The ease of process operation}

In the ECAP process, we have to repeat the operation and the workpiece must experience multiple passes through the conduit in order to achieve a large amount of plastic deformation. While, the increase in the amount of deformation in the HPT process is simply obtained by increasing the number of rotations. Therefore, it is easier to increase the amount of deformation in the HPT process than it is in the ECAP process.

\section{3) Geometry and dimensions of the parts}

When it comes to geometrical forms and dimensions of the parts, a much larger cross sectional area can be accommodated by the ECAP process compared to the HPT process. In the ECAP method, the workpieces could be thin rods, cylindrical parts with a diameter of $20 \mathrm{~mm}$, or even workpieces with square sections. But, since in the HPT process, pressures of several $\mathrm{GPa}$ are applied on the surface of the workpiece, there are size limitations, and in order to achieve such pressures, the researchers usually use small workpieces. On the other hand, as a result of the axisymmetrical nature of the HPT process, the parts produced through this process have an axisymmetrical form which includes discs and rings.

\section{REFERENCES}

[1] R.Z. Valiev, R.K. Islamgaliev, I.V. Alexandrov, “ Bulk nanostructured materials from severe plastic deformation" Prog. Mater. Sci. 45 (2000) 103

[2] A.P. Zhilyaev, T.G. Langdon, "Three-dimensional representations of hardness distributions afterprocessing by high-pressure torsion”Prog. Mater. Sci. 53 (2008) 893.

[3] A.P. Zhilyaev, B.K. Kim, G.V. Nurislamova, M.D. Baró, J.A. Szpunar, T.G. Langdon,"Orientation imaging microscopy of ultrafine-grained nickel" Scripta Mater. 46 (2002) 575.

[4] A.P. Zhilyaev, B.K. Kim, J.A. Szpunar, M.D. Baró, T.G. Langdon, "The microstructural characteristics of ultrafine-grained nickel" Mater. Sci. Eng. A391 (2005) 377.

[5] T. Hebesberger, H.P. Stüwe, A. Vorhauer, F. Wetscher, R. Pippan, "Structure of $\mathrm{Cu}$ deformed by high pressure torsion"
Acta Mater. 53(2005)393.

[6] A.P. Zhilyaev, T.R. McNelley, T.G. Langdon," Evolution of microstructure and microtexture in fcc metals during high pressure torsion” J. Mater. Sci. 42 (2007) 1517.

[7] McFadden SX, Mishra RS, Valiev RZ, Zhilyaev AP, Mukherjee AK. Nature 1999;398:684.

[8] Lu L, Sui ML, Lu K. Science 287:2000:1463.

[9] Zhu YT, Lowe TC, Langdon TG. "Performance and applications of nanostructured materials produced by severe plastic deformation" Scripta Mater 51:2004; 825.

[10] Gleiter H. In: Hansen N, Horsewell A, Leffers T, Lilholt H. Roskilde, Denmark: Riso National Laboratory; 1981. p. 15.

[11] Erb U, El-Sherik AM, Palumbo G, Aust KT." Synthesis, structure and properties of electroplated nanocrystalline materials" Nanostruct Mater 2:1993; 383.

[12] R.Z. Valiev, R.K. Islamgaliev, I.V. Alexandrov, “ Bulk nanostructured materials from severe plastic deformation" Prog. Mater. Sci. 45 (2000) 103.

[13] Valiev RZ, Langdon TG. "Principles of equal channel angular pressing as a processing tool for grain refinement" Prog Mater Sci 51:2006; 881 .

[14] Saito Y, Tsuji N, Utsunomiya H, Sakai T, Hong RG. "Ultra-fine grained bulk aluminum produced by accumulative roll-bonding (ARB) process" Scripta Mater 39:1998; 1221.

[15] Smirnova NA, Levit VI, Pilyugin VI, Kuznetsov RI, Davydova LS, Sazonova VA. Fiz Metal Metalloved 61(6):1986; 1170 .

[16] A.P. Zhilyaev, G.V. Nurislamova , B.-K. Kim , M.D. Baro, J.A. Szpunar, T.G. "Experimental parameters influencing grain refinement and microstructural evolution during high-pressure torsion" Acta Materialia 51 (2003) 753-765

[17] Hall EO. Proc R Soc B 64:1951; 747.

[18] Petch NJ. J Iron Steel Inst 1953;174:25.

[19] Alexander P. Zhilyaev, Terence G. Langdon, "Using high-pressure torsion for metal processing: Fundamentals and applications" Progress in Materials Science 53 (2008) 893-979

[20] R.Z. Valiev, Yu.V. Ivanisenko, E.F. Rauch, B. Baudelet, "Structure and deformaton behaviour of Armco iron subjected to severe plastic deformation" Acta Mater. 44 (1996) 4705 .

[21] F.Wetscher, A. Vorhauer, R. Stock, R. Pippan, "Structural refinement of low alloyed steels during severe plastic deformation" Mater. Sci. Eng. A 387-389 (2004)809.

[22] Zhilyaev AP, Oh-Ishi K, Langdon TG, McNelley TR. "Microstructural evolution in commercial purity aluminum during high-pressure torsion" Mater Sci Eng A, 410-411:2005:277. 